Research Paper

\title{
Aldehyde Dehydrogenase I Immunohistochemical Staining in Primary Breast Cancer Cells Independently Predicted Overall Survival But Did Not Correlate with the Presence of Circulating or Disseminated Tumors Cells
}

\author{
Wendy A. Woodward ${ }^{\bowtie}$, Savitri Krishnamurthy ${ }^{2}$, Ashutosh Lodhi ${ }^{3}$, Lianchun Xiao $^{4}$, Yun Gong ${ }^{2}$, Massimo \\ Cristofanilli ${ }^{5}$, Thomas A. Buchholz ${ }^{1}$, and Anthony Lucci ${ }^{3}$ \\ 1. Departments of Radiation Oncology, The University of Texas M.D. Anderson Cancer Center, Houston, TX, 77030; \\ 2. Departments of Pathology, The University of Texas M.D. Anderson Cancer Center, Houston, TX, 77030; \\ 3. Departments of Surgical Oncology, The University of Texas M.D. Anderson Cancer Center, Houston, TX, 77030; \\ 4. Departments of Biostatistics, The University of Texas M.D. Anderson Cancer Center, Houston, TX, 77030; \\ 5. The Department of Medical Oncology Thomas Jefferson University.
}

\begin{abstract}
$\square$ Corresponding author: Wendy A. Woodward, MD, PhD, wwoodward@mdanderson.org.
(c) Ivyspring International Publisher. This is an open-access article distributed under the terms of the Creative Commons License (http://creativecommons.org/
\end{abstract} licenses/by-nc-nd/3.0/). Reproduction is permitted for personal, noncommercial use, provided that the article is in whole, unmodified, and properly cited.

Received: 2013.10.14; Accepted: 2014.03.19; Published: 20I4.04.07

\begin{abstract}
Purpose: We hypothesized that aldehyde dehydrogenase I (ALDHI) staining in breast cancer tumor cells might be a simple surrogate for the presence of circulating tumor cells (CTCs) or disseminated tumor cells (DTCs).

Experimental Design: Whole tissue primary tumor sections from 121 patients enrolled in a clinical trial assessing CTCs and DTCs at the time of surgery were stained for ALDHI and scored by a dedicated breast pathologist blinded to outcome. Clinical data was extracted and staining was correlated to clinical variables and outcome by Fisher's exact test, the Log rank test and Cox proportional hazards regression analysis respectively. $\mathrm{P}<0.05$ was considered significant.

Results: ALDHI staining in tumor cells was present in $12 \%$ of cases $(15 / 121)$. In univariate analysis, ALDHI tumor staining predicted worse overall survival $(71 \%$ vs. $91 \%$ at 5 years $\mathrm{P}=$ $0.0074)$ and was an independent predictor on multivariable analysis of worse overall survival, (HR 4.93) after adjusting for stage, ER, grade, $L V I$, age and neoadjuvant chemotherapy $(P=0.04)$.

ALDHI was significantly associated with estrogen receptor $(E R)$ negative $(P$ value $=0.029)$ primary tumors but not the presence of CTCs or DTCs by multivariate logistic regression. Positive ALDH staining in non-tumor cells of any pattern or morphology was common but did not correlate with CTCs or DTCs, other clinical variables, or outcome.

Conclusion: ALDHI tumor staining was associated with ER -negative breast cancer and was an independent predictor of OS. However, it did not correlate to putative cancer stem cell surrogates CTCs and/or DTCs.
\end{abstract}

Key words: ALDH1, breast cancer, cancer stem cells, circulating tumor cells, disseminated tumor cells, micrometastatic disease. 


\section{Introduction}

The detection of micrometastatic breast cancer cells in the blood and bone marrow has been correlated with poor outcome [1, 2] suggesting that in some patients these cells are capable of establishing a niche in a distant organ, leading to grossly detectable metastatic disease. It has been suggested that these cells, capable of mediating tumor recurrence from even a single cell, represent cancer stem cells: multipotent, self-renewing cells that might be identifiable by markers or combinations of markers that have been shown to be present on tumor-initiating cells in pre-clinical studies $[3,4]$. We and others have confirmed that putative tumor-initiating or "cancer stem cell" markers can indeed be identified on circulating tumor cells (CTCs) in the blood [5-7] and on epithelial cells in the bone marrow [8]. If further outcome based studies validate the need to eradicate these cells to cure breast cancer, simple, standardized methods to identify patients at risk for micrometastatic disease without the need for a bone marrow biopsy or expensive readout equipment would be desirable.

Aldehyde dehydrogenase (ALDH) 1 is a member of the ALDH superfamily responsible for catalyzing aldehyde oxidation. It has been shown to be expressed in numerous normal tissues including breast epithelium, where it appears to be localized to the myoepithelial cells [4, 9]. ALDH1 staining has been shown by several groups to select for cancer cells capable of enhanced tumorigenesis compared to ALDH1-negative cells [4, 9-11]and several studies have examined the correlation of ALDH1 tumor cell staining with breast cancer prognosis [4, 10-16]. These results have been somewhat mixed likely related to differences in staining technique and scoring, as well as varied size and distribution of clinical variables in patient cohorts examined. Herein, we comprehensively characterize ALDH1 staining in tissue sections from patients with complete clinical information including CTCs and DTCs and report that although ALDH1 staining in primary breast cancers is a strong independent predictor of overall survival which could contribute to a prognostic index incorporating other variables, there was no correlation between ALDH1 and these markers of disseminated micrometastatic disease so it cannot substitute for CTC or DTC assessment when that is deemed appropriate.

\section{METHODS}

Patient data: This cohort represents a subset of patients enrolled on an IRB approved protocol; $\mathrm{Pa}-$ tients with M0 breast cancer had prospective collection of CTCs and DTCs while they were under anesthesia for their primary surgical treatment. Clinical data were retrospectively extracted. Primary tumor tissue available from 121 patients enrolled at the start of the study in 2005 to 2009 was immunostained for ALDH1. Patient and tumor characteristics divided into two groups based on ALDH staining status in tumor cells are listed in Table 1. Twenty Seven patients received anthracycline and taxane based chemotherapy. All Her-2/neu-positive patients received trastuzumab, and estrogen receptor (ER)-positive patients received hormonal therapy. Local regional therapy was breast conserving therapy in 80 patients, and mastectomy with axillary nodal evaluation +/radiation in 41 patients.

Table I: Clinical covariate expression in ALDHI Tumor+ vs. Tumor- patients.

\begin{tabular}{|c|c|c|c|c|}
\hline Covariate & Status & Tumor+ & Tumor- & P-value \\
\hline \multirow[t]{2}{*}{ Nodes+ } & 0 & $59(88.1 \%)$ & $8(11.9 \%)$ & 1.000 \\
\hline & 1 & $47(88.7 \%)$ & $6(11.3 \%)$ & . \\
\hline \multirow[t]{3}{*}{ Stage } & 1 & $36(90 \%)$ & $4(10 \%)$ & 6679 \\
\hline & 2 & $51(87.9 \%)$ & $7(12.1 \%)$ & . \\
\hline & 3 & $19(82.6 \%)$ & $4(17.4 \%)$ & . \\
\hline \multirow[t]{3}{*}{ ER } & 0 & $29(74.4 \%)$ & $10(25.6 \%)$ & $\begin{array}{l}\cdot \\
.0057\end{array}$ \\
\hline & 1 & $77(93.9 \%)$ & $5(6.1 \%)$ & . \\
\hline & & & & . \\
\hline \multirow[t]{3}{*}{ Her2 } & 0 & $100(87.7 \%)$ & $14(12.3 \%)$ & 1.000 \\
\hline & 1 & $6(85.7 \%)$ & $1(14.3 \%)$ & . \\
\hline & & & & . \\
\hline \multirow[t]{3}{*}{ Grade } & 1 or 2 & $65(94.2 \%)$ & $4(5.8 \%)$ & .0231 \\
\hline & 3 & $41(78.8 \%)$ & $11(21.2 \%)$ & . \\
\hline & & & & . \\
\hline \multirow[t]{2}{*}{ LVSI } & 0 & $75(88.2 \%)$ & $10(11.8 \%)$ & 1.000 \\
\hline & 1 & $30(88.2 \%)$ & $4(11.8 \%)$ & . \\
\hline \multirow[t]{2}{*}{ NACT } & 0 & $85(90.4 \%)$ & $9(9.6 \%)$ & .0988 \\
\hline & 1 & $21(77.8 \%)$ & $6(22.2 \%)$ & . \\
\hline \multirow[t]{2}{*}{ death } & 0 & $100(90.1 \%)$ & $11(9.9 \%)$ & .0210 \\
\hline & 1 & $6(60 \%)$ & $4(40 \%)$ & . \\
\hline \multirow[t]{2}{*}{ DTCs } & 0 & $65(92.9 \%)$ & $5(7.1 \%)$ & .0551 \\
\hline & 1 & $17(77.3 \%)$ & $5(22.7 \%)$ & . \\
\hline \multirow[t]{2}{*}{ CTCs } & 0 & $63(86.3 \%)$ & $10(13.7 \%)$ & .5413 \\
\hline & 1 & $32(91.4 \%)$ & $3(8.6 \%)$ & . \\
\hline \multirow[t]{2}{*}{ pCR } & 0 & $16(72.7 \%)$ & $6(27.3 \%)$ & .5552 \\
\hline & 1 & $5(100 \%)$ & $0(0 \%)$ & . \\
\hline
\end{tabular}

Abbreviations: ER, estrogen receptor; LVSI, lymphvascular space in vasion; NACT, neoadjuvant chemotherapy; DTCs, disseminated tumor cells; CTCs, circulating tumor cells; $\mathrm{pCR}$, pathologic complete response. 
Statistics: Categorical variables were tabulated with frequency and percentage. Continuous variables were summarized using descriptive statistics (mean, standard deviation, median and range). The Fisher's exact test and logistic regression analysis were used to determine the association of ALDH expression with the clinical factors. The Kaplan-Meier estimates of survival function, log rank test and Cox proportional hazards regression model were used for the survival analysis.

Immunostaining: Whole tissue sections from the primary tumor of each patient were immunostained by the MDACC clinical core laboratory for ALDH1 (BD Biosciences, Clone 44 dilution 1:100). Positive and negative controls were used with each batch of immunostaining. A pathologist specialized in breast pathology who was blinded to the clinical data reviewed each slide and score the morphology and location of ALDH1+ cells on each slide (Figure 1). Positive cells morphologically resembling histiocytes or lymphocytes were not scored. Positive staining in the tumor (Tumor+) was scored categorically as positive (any positive staining of tumor cells) or negative. Staining of stromal cells within the tumor parenchyma was semiquantitatively scored based on morphology (spindle vs. round, labeled Str_spindle+ vs. Str_round+ respectively) using a 0-3 scale representing frequency of their occurrence. Staining was noted at the periphery of some tumors akin to the "leading edge effect" and this was similarly scored as two variables based on morphology (round vs. spindle shaped, labeled peri_spindle+ vs. peri_round+) with 0-3 scale. In total, five variables were assessed. Frequency of each variable is noted in Table 2.

CTC Analysis: $7.5 \mathrm{~mL}$ of fresh blood was analyzed using the Cellsearch system (Veridex, LLC, Raritan, NJ). Positive CTCs were identified based on staining for DAPI (nuclear stain), CD326 (EpCAM, an epithelial cell surface marker), and the absence of staining for CD45 (white cell marker). Presence of one or more CTCs was considered a positive result.

DTC Analysis: Bone marrow aspirates were obtained from the bilateral iliac crests and pooled for DTC analysis. Enrichment of tumor cells was performed using Ficoll-Hypaque solution. Ten cytospins of the BM were immunostained using a cocktail of pancytokeratin antibodies including CAM5.2 (Beckton Dickinson, San Jose, CA), AE1/AE3 (Dako, Carpinteria, CA), MNF116 (Dako, Carpinteria, CA), cytokeratin 8 (CK8)/ CK18 (Zymed Laboratories, SanFrancisco, CA). A breast cancer pathologist with extensive experience in cytopathology examined each slide and scored DTCs based on cytokeratin positive cells with morphologic characteristics of malignancy.

Table 2: Multivariate logistic regression analysis for association of ALDHI Tumor+ with other clinical factors.

\begin{tabular}{|c|c|c|c|c|}
\hline Effect & Odds Ratio (OR) & $95 \%$ & OR & $P$ value \\
\hline ER (1 vs. 0$)$ & 0.13 & 0.021 & 0.815 & 0.0294 \\
\hline Grade ( 3 vs. 1 or 2$)$ & 3.08 & 0.513 & 18.504 & 0.2187 \\
\hline NACT (1 vs. 0$)$ & 0.602 & 0.114 & 3.174 & 0.5501 \\
\hline DTCs (1 vs. 0$)$ & 1.937 & 0.423 & 8.882 & 0.3946 \\
\hline
\end{tabular}

\section{Results}

\section{ALDHI staining}

The frequency of staining patterns observed in whole tissues is presented in Supplemental Table 1. Tumor+ staining was observed in $12 \%$ of cases, while each of the non-tumor patterns were observed in over $80 \%$ of cases with varying intensity. By Fisher's exact testing, tumor+ staining did not correlate with any of the non-tumor patterns; however all four non-tumor staining patterns were associated with each other. Tumor+ staining was significantly correlated to high tumor grade (11/15 grade 3 among ALDH1 tumor+ vs. 41/106 grade 3 among ALDH1 tumor- ; $P=0.0231$ ) and ER negativity (10/15 ER- among ALDH1 tumor+ vs. $29 / 106$ among ALDH1 tumor-; $\mathrm{P}=0.0057$ ), and trended towards an association with presence of DTCs (5/5 DTC+ among ALDH1 tumor+ vs. 17/82 among ALDH1 tumor- patients; $\mathrm{P}=0.0551)$. Str_round and Str_spindle staining was not significantly correlated with any clinicopathologic variables (including all variables in Table 1). Both Peri_round and Peri_spindle only correlated with node positivity $(P=0.0430)$. Multivariate logistic regression analysis for association of ALDH1 Tumor+ with other clinical factors revealed that ALDH tumor+ staining was independently correlated only to ER-negativity (Table $3)$. Neither the presence of DTCs nor epithelial-defined CTCs was correlated with ALDH+ staining in tumor or stromal cells by logistic regression analysis. 

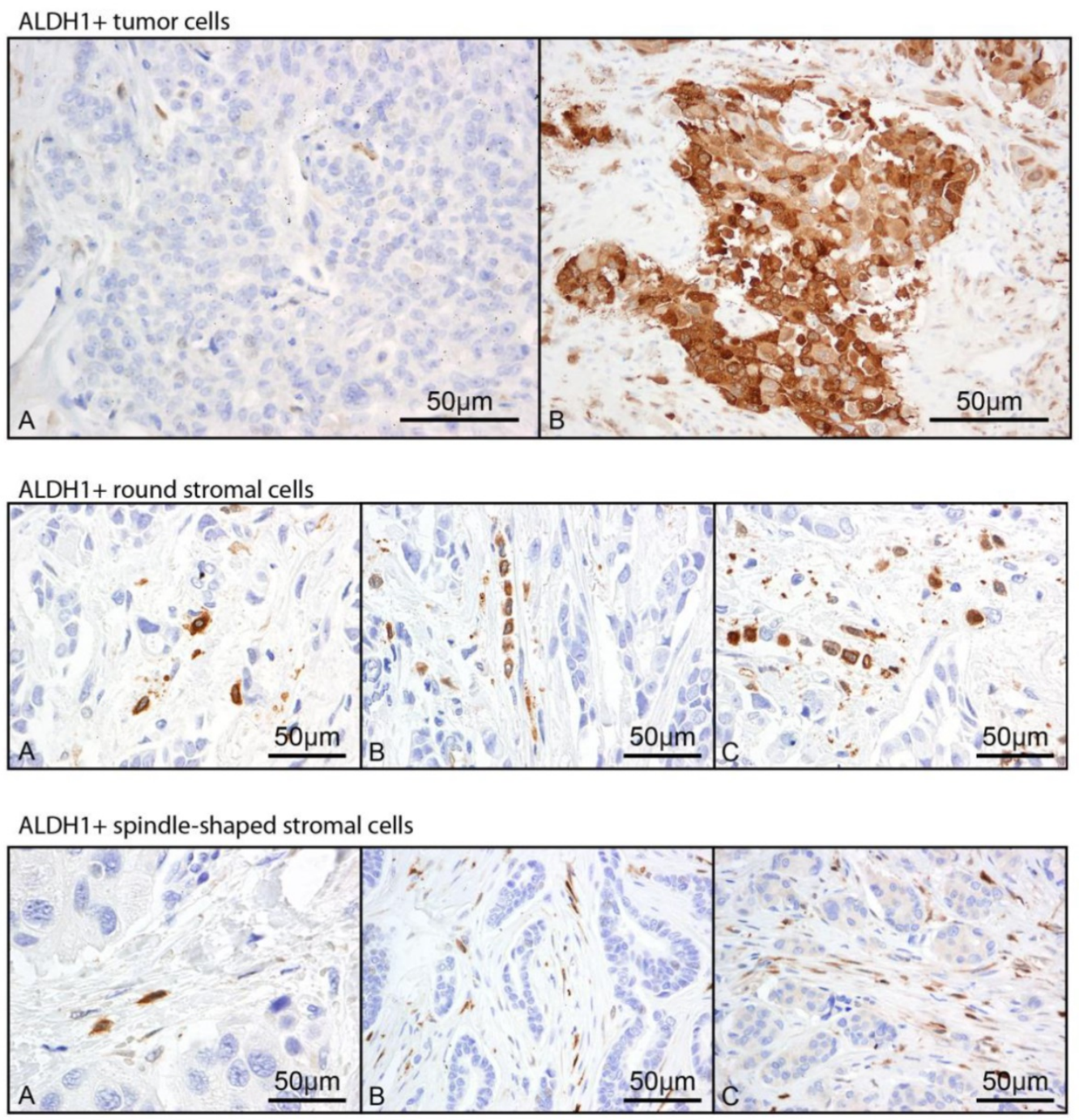

ALDH1+ round cells at the tumor periphery

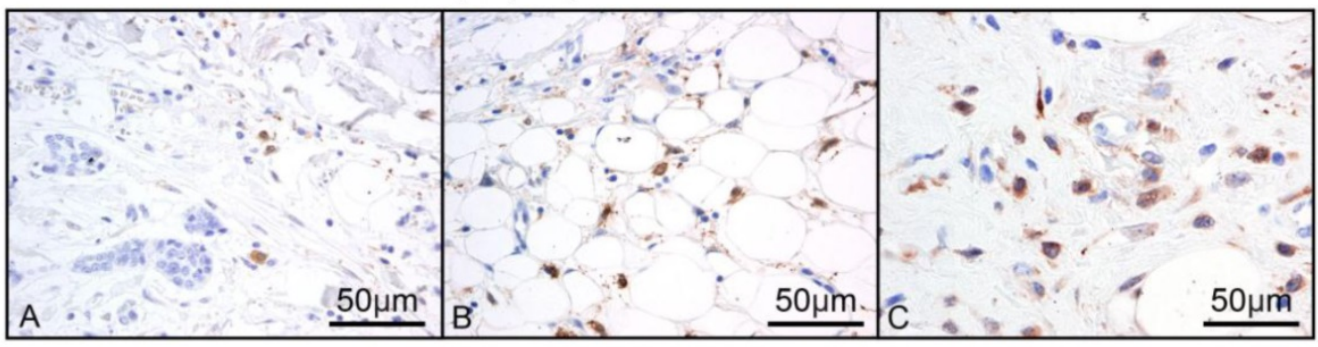

ALDH1+ spindle shaped cells at the periphery

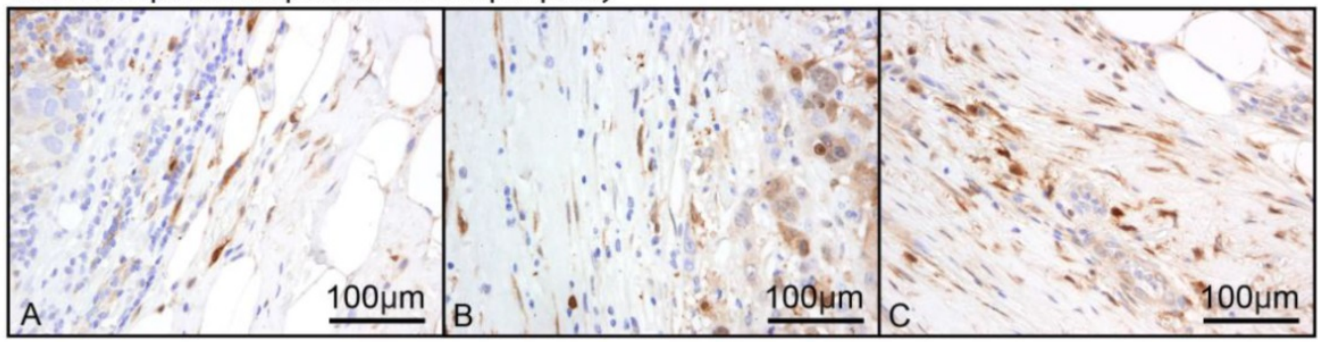

Figure I: Representative images of 5 scored ALDHI staining patterns. Staining in tumor cells (Tumor+) was scored 0 vs. I. Representative images are labeled $A$ and $B$ respectively. Stromal round cells within the tumor parenchyma (Str_round), stromal spindle cells within the tumor parenchyma (Str_spindle), round non-tumor cells at the tumor periphery (Peri_round), and spindle shaped non-tumor cells at the tumor periphery (Peri_spindle) were each scored based on frequency of occurrence from 0-3, with representative images of scores I-3 labeled A-C respectively. 


\section{Overall Survival}

Median follow time was 38 months (interquartile range 27-54 months). Ten patients died of disease. Actuarial 5-yr overall survival was $88.5 \%$. Overall survival was significantly worse in patients with ALDH1 tumor+, advanced stage, use of neoadjuvant chemotherapy (NACT), presence of lymphvascular space invasion (lvsi) and detection of DTCs (Table 3). For example, ALDH expression in tumor was significantly associated with a worse overall survival. The estimated 5-year survival rates for patients with and without ALDH1 Tumor+ staining were 70.6\% (95\% CI: $49.8-100 \%$ ) and $91.3 \%$ (95\% CI: 84.3-98.8\%) respectively (Figure 2A, P- value $=0.0074)$. On multivariate analysis, ALDH1 Tumor+ staining remained an independent predictor of overall survival compared to standard clinicopathologic factors (Table 4 ) with a HR for death of 4.93, $p=0.042$. DTCs were not included in the multivariate model due to missing DTC data in 19 patients (all patients were able to have marrow aspirated). Kaplan-Meier curves for overall survival with ALDH1 tumor+ and/or the presence of DTCs is presented in Figure 2B. Stromal staining was not associated with outcome. Using Fisher's exact test there was no significant association between stromal scores and ER status. However, analysis of each of the four stromal staining variables in the ER- vs. ER+ patients consistently demonstrated a trend for lower overall survival, with increasing intensity of staining among patients with ER-negative disease ( $p$-values for Str_spindle+, Str_round + , peri_spindle+ and peri_round $+0.05,0.05,0.07$, and 0.07 respectively). Given the strong correlation between the four scored variables, all four intensity scores were examined together (any positive vs. negative) for each case and examined in the ER- and ER+ cohort (Figure 2C and 2D). These exploratory data from 39 ER-negative patients suggest stromal staining patterns merit further study in a larger ER-negative patient cohort.

\section{Discussion}

In this study, ALDH1 staining in tumor cells was associated with high grade and ER negative disease, and was an independent predictor for overall survival in patients with non-metastatic, invasive breast cancer. Although ALDH1 is a putative breast cancer stem cell marker identified on epithelial cells found in the blood and bone marrow, here we found no significant correlation between ALDH1 staining in the primary tumor cells and the presence of CTCs or DTCs. If identification of presumed micrometastatic disease in the blood or bone marrow becomes warranted, ALDH1 immunohistochemistry may not a sensitive surrogate for these studies based on the results of this study.

Table 3: Actuarial 5 year overall survival.

\begin{tabular}{|c|c|c|c|c|c|}
\hline Variable & Level & $\mathrm{N}$ & Event & $\begin{array}{l}\text { OS Rate at } 5 \\
\text { Years }(95 \% \mathrm{CI})\end{array}$ & P-value \\
\hline \multirow[t]{2}{*}{ Tumor+ } & 0 & 106 & 6 & $0.913(0.843,0.988)$ & 0.0074 \\
\hline & 1 & 15 & 4 & $0.706(0.498,1)$ & \\
\hline \multirow[t]{4}{*}{ Str_round+ } & 0 & 15 & 1 & $0.833(0.583,1)$ & 0.4846 \\
\hline & 1 & 50 & 2 & $0.947(0.877,1)$ & \\
\hline & 2 & 27 & 4 & $0.804(0.643,1)$ & \\
\hline & 3 & 29 & 3 & $0.89(0.78,1)$ & \\
\hline \multirow[t]{4}{*}{ Str_spindle+ } & 0 & 20 & 1 & $0.857(0.633,1)$ & 0.4326 \\
\hline & 1 & 41 & 2 & $0.938(0.859,1)$ & \\
\hline & 2 & 28 & 4 & $0.782(0.603,1)$ & \\
\hline & 3 & 32 & 3 & $0.895(0.788,1)$ & \\
\hline \multirow[t]{4}{*}{ Peri_round+ } & 0 & 15 & 1 & $0.833(0.583,1)$ & 0.8967 \\
\hline & 1 & 62 & 6 & $0.878(0.788,0.978)$ & \\
\hline & 2 & 21 & 1 & $0.944(0.844,1)$ & \\
\hline & 3 & 20 & 2 & $0.88(0.735,1)$ & \\
\hline \multirow[t]{4}{*}{ Peri_spindle+ } & 0 & 15 & 1 & $0.833(0.583,1)$ & 0.7177 \\
\hline & 1 & 55 & 6 & $0.864(0.765,0.975)$ & \\
\hline & 2 & 28 & 1 & $0.96(0.886,1)$ & \\
\hline & 3 & 20 & 2 & $0.874(0.724,1)$ & \\
\hline \multirow[t]{2}{*}{ Node+ } & 0 & 67 & 3 & $0.913(0.815,1)$ & 0.2048 \\
\hline & 1 & 53 & 6 & $0.864(0.767,0.973)$ & \\
\hline \multirow[t]{3}{*}{ Stage } & 1 & 40 & 2 & $0.886(0.731,1)$ & $<0.0001$ \\
\hline & 2 & 58 & 1 & $0.975(0.928,1)$ & \\
\hline & 3 & 23 & 7 & $0.662(0.484,0.905)$ & \\
\hline \multirow[t]{2}{*}{ ER } & 0 & 39 & 7 & $0.803(0.682,0.946)$ & 0.0171 \\
\hline & 1 & 82 & 3 & $0.922(0.833,1)$ & \\
\hline \multirow[t]{2}{*}{ Her2 } & 0 & 114 & 9 & $0.886(0.813,0.966)$ & 0.7018 \\
\hline & 1 & 7 & 1 & $0.857(0.633,1)$ & \\
\hline \multirow[t]{2}{*}{ Grade } & 1 or 2 & 69 & 4 & $0.896(0.799,1)$ & 0.3087 \\
\hline & 3 & 52 & 6 & $0.871(0.779,0.974)$ & \\
\hline \multirow[t]{2}{*}{ LVSI } & 0 & 85 & 4 & $0.92(0.84,1)$ & 0.0384 \\
\hline & 1 & 34 & 6 & $0.805(0.675,0.959)$ & \\
\hline \multirow[t]{2}{*}{ NACT } & 0 & 94 & 5 & $0.915(0.84,0.995)$ & 0.0328 \\
\hline & 1 & 27 & 5 & $0.792(0.642,0.975)$ & \\
\hline \multirow[t]{2}{*}{ DTCs } & 0 & 70 & 0 & $1(1,1)$ & $<0.0001$ \\
\hline & 1 & 22 & 6 & $0.696(0.517,0.939)$ & \\
\hline \multirow[t]{2}{*}{ CTCs } & 0 & 73 & 6 & $0.863(0.759,0.982)$ & 0.6532 \\
\hline & 1 & 35 & 4 & $0.869(0.757,0.998)$ & \\
\hline \multirow[t]{2}{*}{ pCR } & 0 & 22 & 5 & $0.752(0.584,0.968)$ & 0.2909 \\
\hline & 1 & 5 & 0 & & \\
\hline
\end{tabular}

Abbreviations: ER, estrogen receptor; LVSI, lymphvascular space in vasion; NACT, neoadjuvant chemotherapy; DTCs, disseminated tumor cells; CTCs, circulating tumor cells; $\mathrm{pCR}$, pathologic complete response.

Table 4: Multivariate Cox proportional hazards model for overall survival.

\begin{tabular}{llll}
\hline Variables & & HR $(95 \% \mathrm{CI})$ & P-value \\
\hline Tumor+ & 1 vs. 0 & $4.93(1.06,22.94)$ & 0.042 \\
Stage & 2 vs. 1 & $0.12(0.008,1.81)$ & 0.13 \\
& 3 vs. 1 & $1.65(0.11,25.28)$ & 0.72 \\
ER & 1 vs. 0 & $0.29(0.05,1.77)$ & 0.18 \\
Grade & 2 vs. 1 & $0.09(0.01,0.75)$ & 0.026 \\
& 3 vs. 1 & $0.27(0.02,2.99)$ & 0.29 \\
LVSI & 1 vs. 0 & $6.31(1.12,35.47)$ & 0.037 \\
NACT & 1 vs. 0 & $1.15(0.097,13.54)$ & 0.91 \\
Age & Continuous & $1.04(0.97,1.12)$ & 0.28
\end{tabular}

Abbreviations: ER, estrogen receptor; LVSI, lymphvascular space in vasion; NACT, neoadjuvant chemotherapy. 
A.

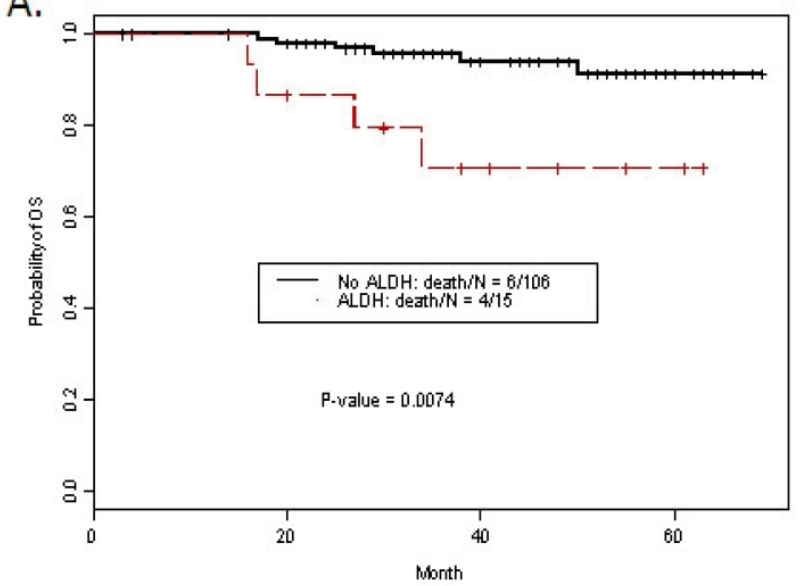

C.

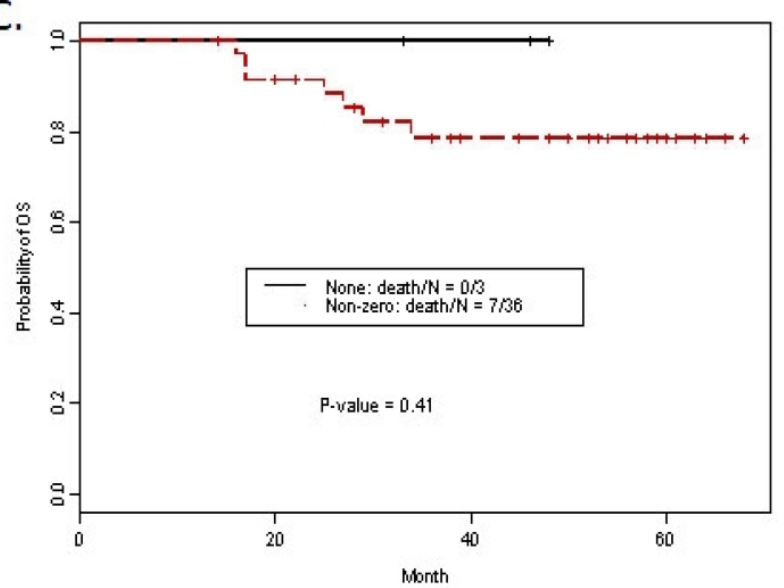

B.

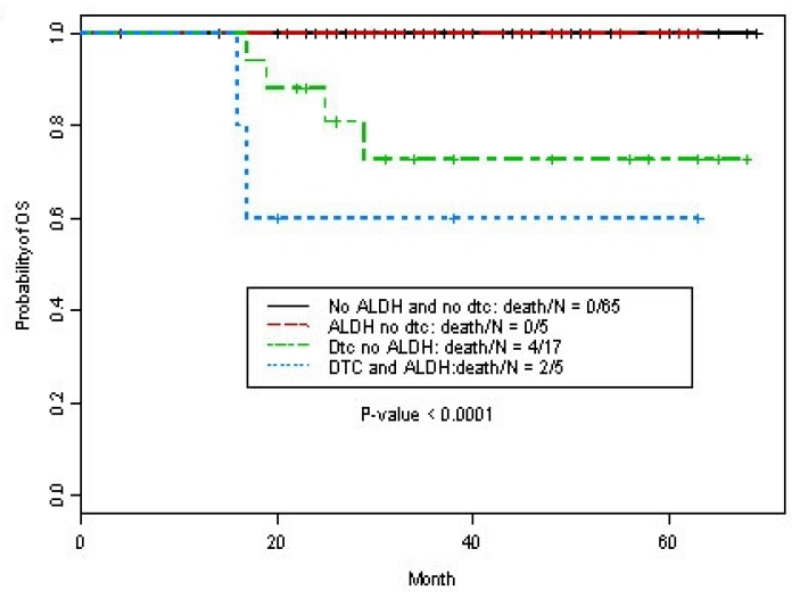

D.

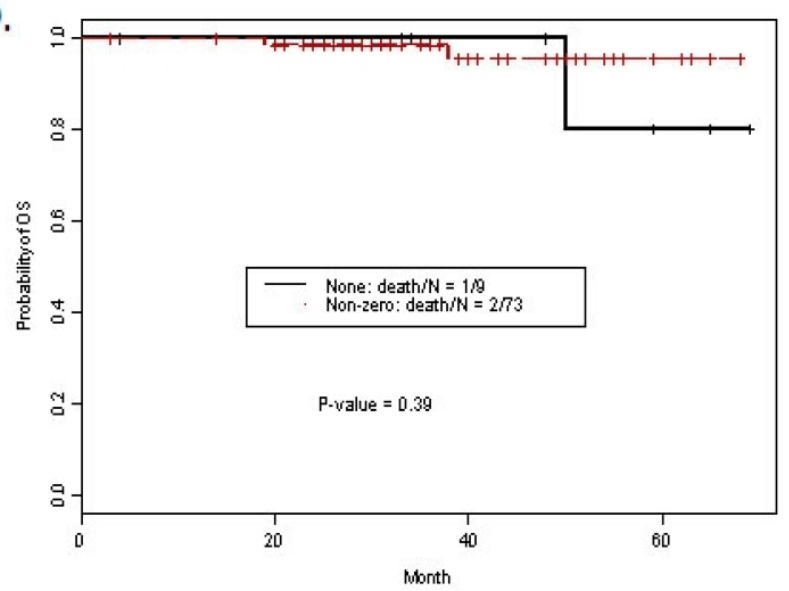

Figure 2: A. Kaplan Meier curves for overall survival by ALDHI staining in tumor cells. B. Kaplan Meier curves for overall survival by ALDHI staining in tumor cells and detection of DTCs. C. Stromal staining (non-zero in any pattern) vs. none in ER negative patients, P = NS. D. Stromal staining (non-zero in any pattern) vs. none in ER positive patients, $\mathrm{P}=\mathrm{NS}$.

It has been hypothesized that cancer stem cells are responsible for micrometastases, recapitulate the original tumor in distant sites, and lead to death from breast cancer. Consistent with this hypothesis we and others have reported that putative breast cancer stem cell markers can be identified on presumed micrometastatic disease [8] using flow cytometry-based approaches that identify ALDH1 enzymatic activity (Aldefluor) in addition to other markers. While some overlap is expected with normal cells (and antibody/aldefluor panels designed to identify epithelial cells in the bone marrow expressing putative cancer stem cell markers also identify a low number of cells in the bone marrow of healthy donors), higher numbers of cells are found in patients at high risk for relapse [8]. Emerging data supports the hypothesis that curing breast cancer requires eradication of micrometastatic breast cancer stem cells. Therefore, a simple immunohistochemistry-based surrogate for micrometastatic disease could greatly impact patient care. Ginestier et al demonstrated that ALDH1 was a tumor-initiating cell marker and validated that immunohistochemical ALDH1 staining in breast cancers was an independent prognostic factor [4]. We hypothesized that ALDH1 staining in primary tumors might represent a surrogate for micrometastatic disease assessed as CTC and DTCs. Using a comprehensive tissue evaluation of staining patterns we confirm the independent prognostic value of ALDH1 staining and outcome, but found no association between ALDH1 tumor staining and micrometastatic disease in this cohort.

The current literature regarding ALDH1 tumor staining as a prognostic marker is varied in patient populations and staining parameters and therefore the results are mixed as well. Consistent findings across reported studies include an association with surrogates for less differentiated tumors including ER negativity, basal subtype, HER2-neu amplification [4, 10, 12]. Herein we confirm higher positivity for ALDH1 tumor staining in patients with ER-negative breast cancer s, as well as higher grade tumors. The 
initial study by Ginestier et al examined ALDH1 tumor staining using two tissue microarrays (TMA) of 134 and 345 patients respectively and found ALDH1 was prognostic on multivariate analysis. ALDH1 staining was present on $19 \%$ and $30 \%$ of tumors respectively. Stromal staining was not examined. A larger TMA-based study examined multiplexed staining from over 600 patients using the AQUA immunofluorescent platform and reported no association between ALDH1 staining and outcome ( $\mathrm{P}=$ 0.09). Without the benefit of histopathology to guide scoring, tumor staining was only considered positive if it co-localized with cytokeratin staining. ALDH1 staining in the CD44+ tumor compartment was noted in only $27 / 490$ cases, but was significantly associated with prognosis on multivariate analysis [13]. Due to the time frame of the patients treated (1962-1983) less than $10 \%$ of this cohort received chemotherapy or endocrine therapy, which may confound the interpretation of this analysis in the setting of contemporary treatment. Multivariate analysis of ALDH1 staining on whole tissue sections was performed by Charaffe-Jauffret et al who reported their findings in 80 patients with inflammatory breast cancer (T4d, stage III) treated from 1976-2003. These authors used non-tumor cells on each slide as an internal control and excluded slides without positive non-tumor cells. They reported that ALDH1 tumor staining was present in $34 \%$ of cases and was prognostic on multivariate analysis [10]. As expected in an inflammatory breast cancer cohort the percentage of ER- negative patients was relatively high at approximately $50 \%$. Morimoto et al examined a cohort of over 200 patients and reported a trend for worse prognosis with high ALDH1 staining $(3+, \mathrm{P}=0.056)$ [12], while Resetkova examined a TMA of 194 cases stained with ALDH1 and found no correlation with outcome [14].

Stromal staining was explicitly excluded from the cohorts described above. Resetkova et al noted that they were unable to assess stromal staining in the $0.6 \mathrm{~mm}$ cores of the TMA but reported on two smaller cohorts of patients with triple receptor negative status, 58 and 40 cases respectively. They found that stromal staining was associated with better outcome. The 40 patient set was used to validate the finding in the first cohort. While it is possible that the unique triple receptor negative cohort accounted for this result, careful scrutiny of the data reveal the stromal staining categories were grouped differently in the two analyses, and the validation would likely have been negative if grouped identically. We have extensively characterized the stromal staining and morphology and find no association with outcome in the total cohort. In light of the above report we performed an exploratory analysis of each stromal variable in the
ER-negative cohort and found no evidence or trend for better outcome among ER-negative patients. On the contrary, for every variable (individually and in all combinations) increasing staining scores were associated with worse outcomes. A larger ER- negative cohort scored in this way would be needed to assess the statistical significance of this observation.

As with most retrospective biomarker studies, this study is limited by the inherent biases associated with retrospective reviews. In addition, until data become clearer regarding clinical or treatment factors that enrich for ALDH1 staining, studies of mixed cohorts are inevitable and may confound the literature. Numerous studies (including ours) include some patients with metaplastic breast cancer, and the percentage of ER-negative patients may be an important factor in the ultimate results. Nonetheless, in this contemporary cohort including clinical stage I-III disease we found that ALDH1 staining was prognostic and independent of known clinical variables. Micrometastatic disease was assessed using current technologies and approaches but is subject to the limitation of the current technology used to identify these cells. Future work may focus on correlating putative cancer stem cells markers to micrometastatic disease identified independent of epithelial markers, since it is hypothesized that micrometastases may lose epithelial markers in the circulation or may stem initially from less differentiated cells.

In conclusion, we report a comprehensive assessment of ALDH1 staining patterns in tumor and stroma using whole tissue sections rather than tissue microarrays. ALDH1 staining in the tumor cells (but not the stroma) was independently associated with poor prognosis and ER negativity, but not micrometastatic disease assessed using epithelial markers.

\section{Supplementary Material}

Supplementary Table 1.

http://www.jcancer.org/v05p0360s1.pdf

\section{Financial support}

The National Institute of Health R01CA138239-01 (WAW, TAB, SK, MC, AL); The State of Texas Grant for Rare and Aggressive Cancers (WAW, TAB, MC, SK, AL); The American Airlines Komen Foundation Promise Grant KGO81287 (WW, SK, AL).

\section{Conflict of Interest}

The authors have no conflicts of interest to disclose. 


\section{References}

1. Braun S, Vogl FD, Naume B, Janni W, Osborne MP, Coombes RC, Schlimok G, Diel IJ, Gerber B, Gebauer G et al: A pooled analysis of bone marrow micrometastasis in breast cancer. N Engl J Med 2005; 353(8):793-802.

2. Cristofanilli M, Budd GT, Ellis MI, Stopeck A, Matera J, Miller MC, Reuben JM, Doyle GV, Allard WJ, Terstappen LW, et al: Circulating tumor cells, disease progression, and survival in metastatic breast cancer. $N$ Engl J Med 2004; 351(8):781-791.

3. Al-Hajj M, Wicha MS, Benito-Hernandez A, Morrison SJ, Clarke MF: Prospective identification of tumorigenic breast cancer cells. Proc Natl Acad Sci U S A 2003; 100(7):3983-3988.

4. Ginestier C, Hur MH, Charafe-Jauffret E, Monville F, Dutcher J, Brown M, Jacquemier J, Viens P, Kleer CG, Liu S, et al: ALDH1 is a marker of normal and malignant human mammary stem cells and a predictor of poor clinical outcome. Cell Stem Cell 2007; 1(5):555-567.

5. Gradilone A, Naso G, Raimondi C, Cortesi E, Gandini O, Vincenzi B, Saltarelli $\mathrm{R}$, Chiapparino E, Spremberg F, Cristofanilli M, et al: Circulating tumor cells (CTCs) in metastatic breast cancer (MBC): prognosis, drug resistance and phenotypic characterization. Ann Oncol, 2010;22(1):86-92.

6. Theodoropoulos PA, Polioudaki H, Agelaki S, Kallergi G, Saridaki Z, Mavroudis D, Georgoulias V: Circulating tumor cells with a putative stem cell phenotype in peripheral blood of patients with breast cancer. Cancer Lett, 2010;288(1):99-106

7. Aktas B, Tewes M, Fehm T, Hauch S, Kimmig R, Kasimir-Bauer S: Stem cell and epithelial-mesenchymal transition markers are frequently overexpressed in circulating tumor cells of metastatic breast cancer patients. Breast Cancer Res 2009;11(4):R46.

8. Reuben JM, Lee BN, Gao H, Cohen EN, Mego M, Giordano A, Wang X, Lodhi A, Krishnamurthy S, Hortobagyi GN et al: Primary breast cancer patients with high risk clinicopathologic features have high percentages of bone marrow epithelial cells with ALDH activity and CD44(+)CD24(lo) cancer stem cell phenotype. Eur I Cancer. 2011;47(10):1527-36.

9. Deng S, Yang X, Lassus H, Liang S, Kaur S, Ye Q, Li C, Wang LP, Roby KF, Orsulic S, et al: Distinct expression levels and patterns of stem cell marker, aldehyde dehydrogenase isoform 1 (ALDH1), in human epithelial cancers. PLoS One, 2010;5(4 e): 10277.

10. Charafe-Jauffret E, Ginestier C, Iovino F, Tarpin C, Diebel M, Esterni B, Houvenaeghel G, Extra JM, Bertucci F, Jacquemier J, et al: Aldehyde dehydrogenase 1-positive cancer stem cells mediate metastasis and poor clinical outcome in inflammatory breast cancer. Clin Cancer Res, 2010; 16(1):45-55.

11. Marcato P, Dean CA, Pan D, Araslanova R, Gillis M, Joshi M, Helyer L, Pan L, Leidal A, Gujar S, et al: Aldehyde dehydrogenase activity of breast cancer stem cells is primarily due to isoform ALDH1A3 and its expression is predictive of metastasis. Stem Cells, 2011;29(1):32-45.

12. Morimoto $K$, Kim SJ, Tanei $T$, Shimazu $K$, Tanji $Y$, Taguchi $T$, Tamaki $Y$, Terada N, Noguchi S: Stem cell marker aldehyde dehydrogenase 1-positive breast cancers are characterized by negative estrogen receptor, positive human epidermal growth factor receptor type 2, and high Ki67 expression. Cancer Sci 2009; 100(6):1062-1068.

13. Neumeister V, Agarwal S, Bordeaux J, Camp RL, Rimm DL: In situ identification of putative cancer stem cells by multiplexing ALDH1, CD44, and cytokeratin identifies breast cancer patients with poor prognosis. Am J Pathol, 2010;176(5):2131-2138.

14. Resetkova E, Reis-Filho JS, Jain RK, Mehta R, Thorat MA, Nakshatri H, Badve S: Prognostic impact of ALDH1 in breast cancer: a story of stem cells and tumor microenvironment. Breast Cancer Res Treat, 2010; 123(1):97-108.

15. Sophos NA, Vasiliou V: Aldehyde dehydrogenase gene superfamily: the 2002 update. Chem Biol Interact 2003; 143-144:5-22.

16. Zhou L, Jiang Y, Yan T, Di G, Shen Z, Shao Z, Lu J: The prognostic role of cancer stem cells in breast cancer: a meta-analysis of published literatures. Breast Cancer Res Treat, 2010;122(3):795-801. 